\title{
Automated multimodal breast CAD based on registration of MRI and two view mammography
}

\author{
T. Hopp, P. Cotic Smole, N.V. Ruiter
}

Karlsruhe Institute of Technology, Institute for Data Processing and Electronics Karlsruhe, Germany

torsten.hopp@kit.edu

\begin{abstract}
Computer aided diagnosis (CAD) of breast cancer is mainly focused on monomodal applications. Here we present a fully automated multimodal CAD, which uses patient-specific image registration of MRI and two-view X-ray mammography. The image registration estimates the spatial correspondence between each voxel in the MRI and each pixel in cranio-caudal and mediolateral-oblique mammograms. Thereby we can combine features from both modalities. As a proof of concept we classify fixed regions of interest (ROI) into normal and suspect tissue. We investigate the classification performance of the multimodal classification in several setups against a classification with MRI features only. The average sensitivity of detecting suspect ROIs improves by approximately $2 \%$ when combining MRI with both mammographic views compared to MRI-only detection, while the specificity stays at a constant level. We conclude that automatically combining MRI and X-ray can enhance the result of a breast CAD system.
\end{abstract}

Keywords: Computer Aided Diagnosis, Multimodal Image Registration, X-ray mammography, MRI

\section{Introduction}

Computer aided diagnosis (CAD) for breast cancer detection has been widely studied in the last years. Most applications of breast CAD have been developed for X-ray mammography [1. Furthermore, CAD has often been applied for breast magnetic resonance imaging (MRI) 2] and breast sonography [3]. While breast CAD is thereby mostly limited to monomodal imaging, several studies, e.g. 4, have shown that the combination of modalities can lead to better detection rates. There are only few approaches combining the diagnostic information of two imaging modalities for breast CAD, e.g. [5. One reason is the challenging spatial correlation of tissue structures as in X-ray mammography, MRI and sonography the patient positioning and compression state of the breast is considerably different. In order to apply a multimodal CAD this leads to manual selection of corresponding tissue structures in multiple modalities [5], which requires experienced radiologists and is time consuming.

In our previous work we developed and evaluated an automated method for MRI to X-ray mammography image registration based on a biomechanical 
model 6. It allows estimating the position of a tissue structure in the X-ray mammogram given its location in the MRI, i.e. for each voxel in the MRI the corresponding pixel in the mammogram is computed. Based on this registration we proposed an automated multimodal CAD approach using the combination of X-ray mammograms and MRI [7. While this first study was limited to cranio-caudal mammograms, we now extended the method to two-view mammograms allowing the automated combination of cranio-caudal mammograms, mediolateral-oblique mammograms and MRI in a CAD system. In this paper we present a proof of concept and give a first estimate of the gain by automatically integrating information of MRI and two-view mammography in breast CAD.

\section{Methods}

\subsection{Image registration}

For automated combination of modalities, the spatial correspondence between MRI and both mammographic views needs to be estimated. We apply an image registration which uses a biomechanical model of the breast to simulate the mammographic compression. The patient-specific biomechanical model is generated from the segmented MR volume. For the segmentation, a fuzzy C-means clustering similar to 8 and edge detection is applied. The model geometry is assembled by a tetrahedral mesh differentiating fatty and glandular tissue. Both tissue types are modeled as hyperelastic neo-hookean material with individual material parameters for fatty and glandular tissue. Mammographic compression is simulated by adding compression plates into the simulation and formulating a contact problem which is solved by the Finite Element method and solved using the dynamic solver of the software package ABAQUS. Based on the estimated deformation field, each three-dimensional point in the MR volume can be mapped to a two-dimensional point in the X-ray mammogram. This is achieved by simulating a perspective X-ray casting on the deformed MRI. The registration was carried out for both mammographic views, i.e. cranio-caudal (CC) and mediolateral-oblique (MLO) mammograms. For more details refer to our earlier publications [69.

\subsection{CAD system}

As a proof of concept, we designed a CAD system which aims to classify distinct cubic regions of interest (ROIs) into one of the categories normal or suspect. For this purpose, the breast in the MR volume is quantized into ROIs of size $(10 \times 10 \times 10) \mathrm{mm}^{3}$. To extract multimodal information, the eight vertices of the MRI ROIs are mapped to the CC mammogram as well as the MLO mammogram based on the deformation fields computed during the image registration. The mammography ROIs are then formed by the convex hull of these eight automatically mapped points (Figure 1).

For each 3D MRI ROI, 64 features are extracted. Intensity based features include e.g. the mean intensity and its variance at three time points (pre-contrast, 


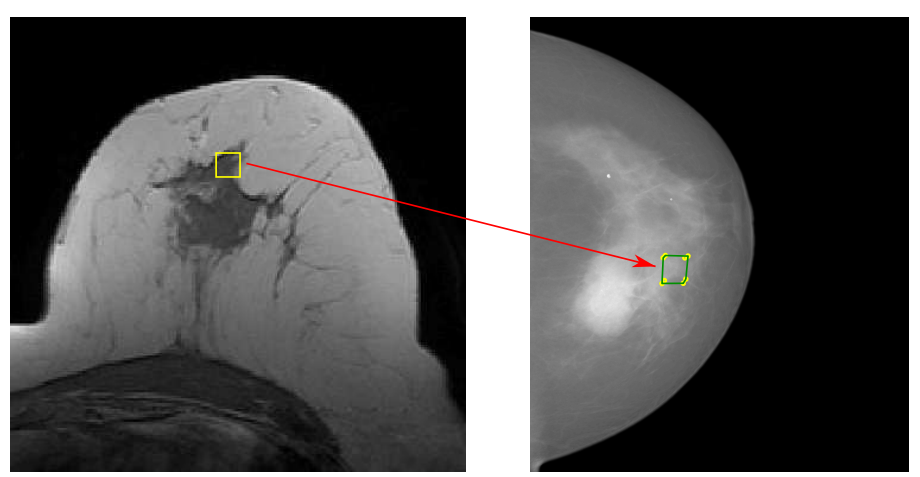

Fig. 1. Mapping from the MRI (left) to the CC mammogram (right) based on the estimated deformation field. The vertices of the MRI ROI (yellow rectangle) are mapped to the X-ray mammogram (yellow dots). The mammography ROI is formed by the convex hull of these points (green).

$1 \mathrm{~min}$ and 6-7 min post-contrast). Texture features are based on 3D gray-level co-occurence matrices [10. Temporal features analyze the contrast enhancement from pre-contrast to $1 \mathrm{~min}$ post-contrast and from $1 \mathrm{~min}$ post-contrast to the last time point similar to the three time points method [11.

For each mapped mammography ROI, 54 features are extracted. Similar to the MRI intensity features, e.g. the mean intensity and its median and variance in the ROI are computed. Texture features are based on gray-level co-occurence matrices [12] and gray-level runlength matrices [13. Furthermore a multilevel Otsu thresholding [14] is applied and features based on morphological enhancement [15] are added. All features from MRI and mammography are gathered in a combined feature vector. Combining the MRI features with CC and MLO mammography features, a total of up to 172 features are used to classify each ROI.

The classification problem is addressed by the WEKA pattern recognition toolbox [16. A correlation based feature subset selection is performed with a best-first search method 17 using a ten-fold cross-validation. Features selected in at least one of ten folds were then selected for classification. We repeated the cross-validation for the feature selection three times with varying random seed in order to reduce the sensibility of our analysis due to the dataset splitting.

To demonstrate the feasibility of the approach, we applied a random forest classifier as it proved to provide robust results in our earlier study [7]. The classifier performance was again evaluated by a ten-fold cross-validation and repeated three times with a random seed.

\subsection{Clinical datasets and evaluation methods}

The method was evaluated using 43 patient datasets from a previous study for which an image registration of MRI with CC as well as with the MLO oblique 
mammogram was carried out 618. Each dataset included a time series of T1weighted dynamic contrast enhanced MR volumes and the corresponding CC and MLO mammogram of the same patient. MR images were acquired on 1.5 Tesla scanners (Siemens Magnetom Symphony, Sonata, Avanto) with the patient in prone position using dedicated bilateral breast coils. The MRI parameters were as follows: matrix size $=384 \times 384$, slices $=33$, spatial resolution $=$ $0.9 \times 0.9 \times 3.0 \mathrm{~mm}^{3}$, time of acquisition $=1 \mathrm{~min}$. per measurement. As a contrast agent, $0.1 \mathrm{mmol} /(\mathrm{kg}$ body weight) gadopentetate-dimeglumine (Gd-DTPA) was administered at $3 \mathrm{ml} / \mathrm{s}$ intravenously using a power injector for standardized injection. The contrast agent injection bolus was followed by $20 \mathrm{ml}$ of physiological saline solution. Full field digital mammograms were acquired on GE Senograph 2000D units.

The ground truth for the classifier training and evaluation is given based on expert annotations. Each annotation circumscribes the lesion in the 3D MR volume with a freehand tool. The annotated lesions included a mixture of malign and benign lesions verified by histology or follow-up diagnosis. A ROI of our CAD approach was labeled as suspicious if at least $50 \%$ of its volume was covered by the expert's lesion annotation.

As a proof of principle, a ROI size in the MRI of $10 \times 10 \times 10 \mathrm{~mm}^{3}$ was chosen as a tradeoff between detectable lesion size and the time consumption for mapping the ROI from MRI to both mammographic views. This resulted in a total of 31,239 ROIs of which 634 were labeled as suspicious.

The image registration of these datasets was performed in previous studies [69]. The target registration error (TRE) was estimated based on the Euclidean distance between the annotated lesion center in the mammogram and the annotated lesion center projected from the 3D MRI into the 2D mammogram. The average TRE was approximately $13.6 \mathrm{~mm}$ (Standard deviation (SD) $9.6 \mathrm{~mm}$ ) 6 for the $\mathrm{CC}$ view mammograms and $16.3 \mathrm{~mm}$ (SD $8.7 \mathrm{~mm}$ ) for the MLO view mammograms.

For the evaluation we tested our CAD system with four different setups: 1) using only features from MRI, 2) using features from MRI and CC-view mammograms, 3) using features from MRI and MLO-view mammograms, 4) using features from MRI and CC- and MLO-view mammograms. For each setup the previously described feature selection and classifier evaluation by ten-fold cross-validation was carried out. Hence, in total we obtained nine classification evaluation runs per setup with all combinations of three subsets of the features and three random seeds for the classifier cross-validation. To analyze the classifier performance, the error rate of incorrectly classified ROIs as well as the ROI related sensitivity and specificity were calculated from the true and false positive respectively true and false negative rates, where suspicious ROIs are positive.

\section{Results}

Figure 2 and 3 show box plots of the evaluation of the four setups. The average sensitivity increases from an average of $76.1 \%$ ( \pm standard deviation $0.8 \%$ ) if only 


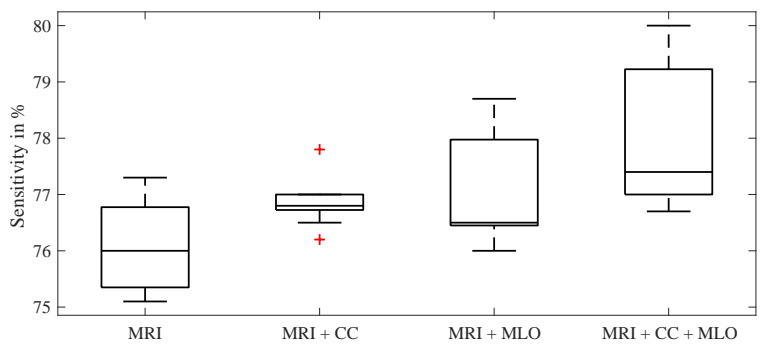

Fig. 2. Classification result: sensitivity of detecting suspicious ROIs with the evaluated four setups. The tops and bottoms of each "'box"' are the 25 th and 75 th percentiles of the samples respectively, the line within the box gives the median value. The vertical dashed lines indicate the whiskers which include all datasets not considered as outliers, i.e. are below 1.5 times the interquartile range.

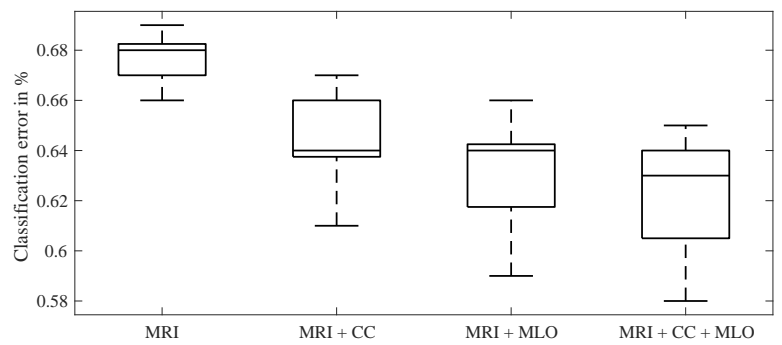

Fig. 3. Classification result: percentage of wrongly classified ROIs with the evaluated four setups. The tops and bottoms of each "'box"' are the 25 th and 75 th percentiles of the samples respectively, the line within the box gives the median value. The vertical dashed lines indicate the whiskers which include all datasets not considered as outliers, i.e. are below 1.5 times the interquartile range.

MRI features are considered (setup 1) to $76.9 \%( \pm 0.4 \%), 77.1 \%( \pm 1.0 \%)$ and $77.9 \%( \pm 1.2 \%)$ for the multimodal setups $2(\mathrm{MRI}+\mathrm{CC}), 3(\mathrm{MRI}+\mathrm{MLO})$ and $4(\mathrm{MRI}+\mathrm{CC}+\mathrm{MLO})$ respectively. At the same time the ROI related specificity stays at a constant level of $99.8 \%$ for all setups. This trend is also present in the error rate of wrongly classified ROIs: it decreases from $0.68 \%$ (MRI) to $0.64 \%$ $(\mathrm{MRI}+\mathrm{CC}), 0.63 \%(\mathrm{MRI}+\mathrm{MLO})$ and $0.62 \%(\mathrm{MRI}+\mathrm{CC}+\mathrm{MLO})$. Hence the best result is obtained by combining the multimodal information from MRI and both mammographic views.

In order to analyze the influence of the TRE on the classification performance, we selected a subset of all datasets with the TRE below $10 \mathrm{~mm}$ in both the $\mathrm{CC}$-view registration and the MLO-view registration. Thereby the TRE equals the size of a ROI in our evaluated scenario, which ensures that the true lesion 
positions in both mammographic views overlap with the mapped ROIs in all cases.

For this data subset the same evaluation as before was carried out. The sensitivity for setup 1, 2, 3 and 4 was $76.2 \%( \pm 4.2 \%), 79.6 \%( \pm 3.2 \%), 75.7 \%$ $( \pm 4.2 \%)$ and $78.3 \%( \pm 3.5 \%)$. At the same time the specificity changes only slightly and increases by adding multimodal information: for setup 1,2,3 and 4, the average specificities are $99.6 \%( \pm 0.1 \%), 99.7 \%( \pm 0.1 \%), 99.7 \%( \pm 0.1 \%)$ and $99.7 \%( \pm 0.1 \%)$. The according classification errors are $0.81 \%( \pm 0.11 \%), 0.69 \%$ $( \pm 0.12 \%), 0.83 \%( \pm 0.05 \%)$ and $0.62 \%( \pm 0.02 \%)$.

Adding information from $\mathrm{CC}$ view mammograms increases the sensitivity considerably more than in the evaluation with all datasets. Yet, in the MLO case the sensitivity slightly decreases, while in combined CC-view and MLOview case, intermediate results can be observed. The same holds for the classification error. The specificity remains at an approximately constant level for all multimodal setups. One reason for the mixed results might be the limited size of the data subset: it consists of three patient datasets only with a TRE below $10 \mathrm{~mm}$ which equals a total of 2195 ROIs. This hypothesis is supported by the considerably larger standard deviations compared to the evaluation with all datasets.

\section{Discussion and Conclusion}

In this paper we presented a method for an automated multimodal CAD system based on an image registration of 3D MRI and 2D two-view X-ray mammography. We extend our earlier approach by adding MLO mammograms into the CAD system and analyzed the improvement by combining multimodal information in a classical pattern recognition for breast cancer detection.

To check the feasibility of our approach, we used distinct ROIs with a size of $10 \times 10 \times 10 \mathrm{~mm}^{3}$. Due to the fixed ROI size and the availability of only freehand annotations of lesions, the labeling of each ROI was not straight forward. We decided initially for a labeling of the ROIs based on their overlap with the lesion annotation and used a volume overlap threshold of $50 \%$ to label the ROI as suspicious. Thereby the lesion size that can be detected by our system is limited. Our current research focuses on the one hand on acceleration of the mapping between MRI and mammography to allow a higher number and hence a smaller size of ROIs or even a voxel based classification using a sliding window approach. On the other hand the influence of the labeling threshold will be investigated further and we are planning an additional review of the labeling with experts to clean the dataset from outliers.

Similar to our earlier study, this work is limited to datasets selected retrospectively from clinical routine where all MRI examinations were carried out with the same protocol. Mammograms were all acquired with the same mammography system leading to homogeneous image characteristics. Datasets were furthermore selected such that a lesion could be delineated in both the MRI and X-ray mammogram by an expert in order to evaluate the TRE. 
For evaluation of our CAD approach, a basic set of commonly used features was extracted for MRI and both mammographic views. This might not yet tap the full potential of a CADe system, yet we allow easy extension of the system in future by a plugin-like feature extraction software architecture.

Despite the limitations of this study, the initial results are promising: Approximately $78 \%$ of the suspect labeled ROIs could be identified by our proposed method. Though the average registration error was larger than the ROI size, the results improved when combining multimodal information. Due to non-linear deformations, complex tissue structures and manual interactions during the patient positioning, the accurate registration of X-ray mammograms and MRI is challenging and a lively field of research. Yet a TRE in the range of current MRI to mammography registration approaches [6]19|20 already leads to improvements of the CADe performance. We showed in this study that including a second mammographic view can further enhance our multimodal CAD system for breast cancer detection.

\section{References}

1. Cheng, H., Shi, X., Min, R., Hu, L., Cai, X., Du, H.: Approaches for automated detection and classification of masses in mammograms. Pattern Recognition 39(4) (2006) 646-668

2. Dorrius, M., der Weide, M.v., van Ooijen, P., Pijnappel, R., Oudkerk, M.: Computer-aided detection in breast MRI: a systematic review and meta-analysis. European Radiology 21(8) (2011) 1600-1608

3. Cheng, H., Shan, J., Ju, W., Guo, Y., Zhang, L.: Automated breast cancer detection and classification using ultrasound images: A survey. Pattern Recognition 43(1) (2010) 299-317

4. Lord, S., Lei, W., Craft, P., Cawson, J., Morris, I., Walleser, S., Griffiths, A., Parker, S., Houssami, N.: A systematic review of the effectiveness of magnetic resonance imaging (MRI) as an addition to mammography and ultrasound in screening young women at high risk of breast cancer. European Journal of Cancer 43(13) (2007) 1905-1917

5. Yuan, Y., Giger, M.L., Li, H., Bhooshan, N., Sennett, C.A.: Multimodality computer-aided breast cancer diagnosis with FFDM and DCE-MRI. Academic Radiology 17(9) (2010) 1158-1167

6. Hopp, T., Dietzel, M., Baltzer, P., Kreisel, P., Kaiser, W., Gemmeke, H., Ruiter, N.: Automatic multimodal $2 \mathrm{D} / 3 \mathrm{D}$ breast image registration using biomechanical FEM models and intensity-based optimization. Medical Image Analysis 17(2) (2013) 209-218

7. Hopp, T., Neupane, B., Ruiter, N.V.: Automated multimodal computer aided detection based on a $3 \mathrm{~d}-2 \mathrm{~d}$ image registration. In: Proceedings 13th International Workshop on Breast Imaging, IWDM 2016, Malmö, Sweden,. (2016) 400-407

8. Wu, S., Weinstein, S., Keller, B., Conant, E., Kontos, D.: Fully-automated fibroglandular tissue segmentation in breast MRI. In: IWDM 2012, LNCS 7361. (2012) $244-251$

9. Hopp, T., de Barros Rupp Simioni, W., Perez, J.E., Ruiter, N.: Comparison of biomechanical models for MRI to X-ray mammography registration. In: Proceedings 3rd MICCAI Workshop on Breast Image Analysis. (2015) 81-88 
10. Chen, W., Giger, M.L., Li, H., Bick, U., Newstead, G.M.: Volumetric texture analysis of breast lesions on contrast-enhanced magnetic resonance images. Magnetic Resonance in Medicine 58(3) (2007) 562-571

11. Degani, H., Gusis, V., Weinstein, D., Fields, S., Strano, S.: Mapping pathophysiological features of breast tumors by MRI at high spatial resolution. Nature Medicine 3(7) (1997) 780-782

12. Haralick, R.M., Shanmugam, K., Dinstein, I.: Textural features for image classification. IEEE Trans. Systems, Man, and Cybernetics SMC-3(6) (1973) 610-621

13. Galloway, M.M.: Texture analysis using gray level run lengths. Computer graphics and image processing $4(2)$ (1975) 172-179

14. Otsu, N.: A threshold selection method from gray-level histograms. IEEE Trans. Systems, Man, and Cybernetics 9(1) (1979) 62-66

15. Li, H., Wang, Y.J., Liu, K.J.R., Lo, S.C.B., Freedman, M.T.: Computerized radiographic mass detection - part i: Lesion site selection by morphological enhancement and contextual segmentation. IEEE Trans. Medical Imaging 20 (2001) 289-301

16. Hall, M., Frank, E., Holmes, G., Pfahringer, B., Reutemann, P., Witten, I.H.: The WEKA data mining software: An update. SIGKDD Expl. 11(1) (2009) 10-18

17. Hall, M.A.: Correlation-based Feature Subset Selection for Machine Learning. PhD thesis, The University of Waikato, Hamilton, New Zealand (1999)

18. Hopp, T., Ruiter, N.V.: 2d/3d registration for localization of mammographically depicted lesions in breast mri. In: Proceedings 11th International Workshop on Breast Imaging, IWDM 2012, Philadelphia, PA, USA. (2012) 627-634

19. Mertzanidou, T., Hipwell, J., Johnsen, S., Han, L., Eiben, B., Taylor, Z., Ourselin, S., Huisman, H., Mann, R., Bick, U., Karssemeijer, N., Hawkes, D.: MRI to xray mammography intensity-based registration with simultaneous optimisation of pose and biomechanical transformation parameters. Medical Image Analysis 18(4) (2014) 674-683

20. Lee, A., Rajagopal, V., Gamage, T.P.B., Doyle, A.J., Nielsen, P., Nash, M.: Breast lesion co-localisation between X-ray and MR images using finite element modelling. Medical Image Analysis 17(8) (2013) 1256-1264 